\title{
PROBABILITY OF RUIN UNDER INFLATIONARY CONDITIONS OR UNDER EXPERIENCE RATING
}

\author{
G. C. TAYLOR*
}

The effect of inflation of premium income and claims size distribution, but not of free reserves, on the probability of ruin of an insurer is studied.

An interesting similarity between this problem and the ruin problem in an experience-rated scheme is exhibited. This similarity allows the deduction of parallel results for the two problems in later sections.

It is shown that the probability of ruin is always increased when the (constant) inflation rate is increased.

The distribution of aggregate claims under inflationary conditions is described and used to calculate an upper bound on the ruin probability. Some numerical examples show that this bound is not always sharp enough to be practically useful. It is also shown, however, that this bound can be used to construct an approximation of the effect of inflation on ruin probability.

It is shown that if inflation occurs at a constant rate, then ruin is certain, irrespective of the smallness of that rate and of the largeness of initial free reserves and the safety margin in the premium. The corresponding result for experiencerated schemes is that a practical and "intuitively reasonable" experience-rating scheme leads eventually to certain ruin.

Finally, a simple modification of the techniques of the paper is made in order to bring investment income into account.

\section{INTRODUCTION}

The probability of ruin of a risk business has been studied under various conditions in the past, e.g. Lundberg (1909), Cramér (1930, 1955), and others. Most of these studies have assumed that the risk process is either a stationary one or can be made stationary by means of a simple transformation.

Such models of the risk process do not include the case in which the phenomenon of inflation is causing the volume of premium income and of claims but not free reserves to vary in time. In current times, when rates of inflation in many countries have been, are and appear likely to remain for some time at high levels, it seems advisable to examine the impact of this feature on the solvency of the risk business in so far as this latter is described by the probability of ruin.

In carrying out this examination, it is noted that the operation of certain types of experience rating schemes is closely parallel to that of inflation on a "conventional" risk business, so that the methods foreshadowed in the preceding paragraph are also applicable to experience rated processes.

* The author gratefully acknowledges the use of facilities of the Swiss Reinsurance Company, Zurich, Switzerland in the preparation of this paper. 


\section{DESCRIPTION OF THE RISK PROCESS}

We consider a risk process in which premiums received in the time-interval $[0, t]$ total $C(t)$ (the process begins at $t=0$ ). Let $X(t)$ denote the aggregation of claims occurring in the time-interval $[0, t]$.

Suppose that $\{X(t), t \geqslant 0\}$ is a one-dimensional Markov process. Let $Z(t)$ denote the free reserves at time $t$ and write $x$ for $Z(0)$. Then

$$
Z(t)=x+C(t)-X(t)
$$

is also a one-dimensional Markov process.

Since $X(t)$ is the aggregation of claims up to time $t$, it is possible to write

$$
X(t)=\sum_{i=1}^{N(t)} S_{i}
$$

where $S_{i}$ is the random variable denoting the size of the $i$-th claim and $N(t)$ is the random variable denoting the number of claims occurring in the time-interval $[0, t]$.

Sometimes in the following sections, no further assumptions about the risk process will be made. At other times it will be necessary to place some restrictions on the random variables $N(t)$ and $S_{i}$.

\section{ADDITION OF INFLATION TO THE RISK PROCESS}

We now wish to superimpose an inflation process on the risk model described in Section 2. We suppose this process to be a deterministic one in that we assume the existence of a non-stochastic inflation factor $f(t)(>0)$ at time $t$.

Premium volume at time $t$ and also claims paid at time $t$ are inflated by the factor $f(t)$ (assume $f(0)=1$ ). Let $C^{*}, X^{*}$ and $Z^{*}$ represent the functions $C$, $X$ and $Z$ respectively after modification by the factor $f$. Then

$$
\begin{aligned}
C^{*}(t) & =\int_{0}^{t} f(s) d C(s) \\
X^{*}(t) & =\int_{0}^{t} f(s) d X(s) \\
& =\sum_{i=1}^{N(t)} f\left(t_{i}\right) S_{i},
\end{aligned}
$$

where $t_{i}$ is the epoch of the $i$-th claim;

$$
Z^{*}(t)=x+C^{*}(t)-X^{*}(t) .
$$

Note that in (4) inflation is assumed to have no effect on free reserves. This is not unrealistic in the light of the experience of the last few years. In any case, this restriction is relaxed in Section 12. 
4. THE RELATION BETWEEN INFLATION AND EXPERIENCE RATING

Consider a risk business subject to the same risk process as (1) except that each element of premium paid is modified by a refund or surcharge according to the difference between past premiums and past claims. Suppose that the precise form of this experience rating is such that the element of premium payable at time $t$ is:

$$
d C(t)=\{c-k[C(t)-X(t)]\} d t
$$

$c$ being the base rate of premium payable, i.e. the premium rate when the experience follows its expected pattern exactly; and $k$ being the experience rating factor at time $t$ (normally, $0<k<1$ ).

It is easy to deduce from (5) that

$$
C(t)=\frac{c}{k}\left[1-e^{-k t}\right]+\sum_{i=1}^{N(t)} S_{i}\left[1-e^{-k\left(t-t_{i}\right)}\right],
$$

whence

$$
Z(t)=x+e^{-k t} \int_{0}^{t} c e^{k s} d s-e^{-k t} \sum_{i=1}^{N(t)} S_{i} e^{k t_{i}}
$$

or

$$
\hat{Z}(t)=e^{k t} Z(t)=x e^{k t}+\int_{0}^{t} c e^{k s} d s-\sum_{i=1}^{N(t)} S_{i} e^{k t_{i}}
$$

From (2), (3) and (4) it can be seen that $\hat{Z}(t)$ represents a "conventional" risk process subject to inflation at a continuous rate of $k$ per unit time except that the initial free reserve also inflates at this rate instead of remaining constant as assumed in Section 3.

In each of the following sections, this relation between a risk process in inflationary conditions and an experience-rated risk process permits the deduction of parallel results, although the emphasis is on the former in the section headings.

\section{PROBABILITY OF RUIN IS NONDECREASING WITH INCREASING INFLATION}

This result is proved by showing that any realization of $\left\{Z^{*}(t), t \geqslant 0\right\}$ leading to ruin also leads to ruin if the rate of inflation is increased.

Consider two $Z^{*}$-processes called $Z_{1}^{*}$ and $Z_{2}^{*}$ with associated inflation factors of $f_{1}$ and $f_{2}$ respectively. Suppose a particular realization of $Z_{1}^{*}$ leads to ruin. Then for some $t$, we have

$$
Z_{1}^{*}(t)<0, Z_{1}^{*}(s) \geqslant 0 \text { for } 0 \leqslant s<t .
$$

Now, from (2), (3) and (4),

$$
Z_{2}^{*}(t)-Z_{1}^{*}(t)=\int_{0}^{t}\left[f_{2}(s)-f_{1}(s)\right] d Z(s) .
$$


Integration by parts yields:

$$
Z_{2}^{*}(t)-Z_{1}^{*}(t)=g(t) Z_{1}^{*}(t)-\int_{0}^{t-0} Z_{1}^{*}(s) d g(s)
$$

where $g(s)=f_{2}(s) / f_{1}(s)-1$, and it has been assumed that this function is measurable. If $g(s)$ is a monotone nondecreasing function (recall that $g(0)=0$ ), then $g(s) \geqslant 0$ for $s \geqslant 0$ and $d g(s) \geqslant 0$ for $s \geqslant 0$, and by (8) and (9)

$$
Z_{2}^{*}(t)-Z_{1}^{*}(t) \leqslant 0 \text {. }
$$

We may summarize the above in the following:

\section{Result}

If two $Z^{*}$-processes, $Z_{1}^{*}$ and $Z_{2}^{*}$, are subject to measurable inflation factors of $f_{1}(t)$ and $f_{2}(t)$ such that the difference $f_{2}(t) / f_{1}(t)$ is nondecreasing with increasing $t$, then the probability of ruin (in finite or infinite time) is not less for the $Z_{2}^{*}$-process than for the $Z_{1}^{*}$-process.

\section{Remarks}

I. It is of course assumed that the initial reserves are the same in the $Z_{1^{-}}^{*}$ and $Z_{2}^{*}$-processes.

2. The result is entirely independent of the properties of the process $Z$. It includes, for example, cases where the claim number process is not Poisson, where sizes of different claims are not independent, etc.

3. The requirement that $f_{2}(t) \mid f_{1}(t)$ be monotone nondecreasing is easily seen to be equivalent to the requirement that the $Z_{2}^{*}$-inflation rate should always be not less than the $Z_{1}^{*}$-inflation rate in those cases where $f_{1}$ and $f_{2}$ are smooth and the term "inflation rate" therefore meaningful.

The situation for the experience-rated process $\hat{Z}$ is not so simple, However, in the case of zero initial reserves (i.e. $x=0$ ), we see from a comparison of equations (4) and (7) that the $\hat{Z}$-process is exactly the same as a $Z^{*}$-process with $f(t)=\exp (k t)$. It follows, therefore, that, in this case, increasing $k$, the degree of experience rating, will increase the ruin probability.

We shall see further, in Section 11, that under experience rating the ultimate $(t=\infty)$ probability of ruin is always 1 .

That these results are not intuitive to some extent is clear from a paper by SEAL (I969), in which he refers to the criticism that his simulated ruin probabilities (according to "conventional" risk processes) were too high. The suggestion is that in practice an insurer can use some kind of experience rating and, by basing premiums on past results, will be able to reduce the ruin probability.

The reasoning leading to this conclusion is probably somewhat along the 
following lines. There are two important classes of free reserve trajectory: that consisting of trajectories characterized by persistently light claims experience, and that characterized by persistently heavy claims experience. In the first case ruin does not occur whether experience-rated or not; in the second, premium rates are forced up by the poor experience, thus reducing the proportion of ruins.

The fallacy in such an argument is that it ignores the possibility of a light claims experience followed by a slightly heavier than usual experience. In this case the initial light experience forces premiums down so that the fund built up in this period is not particularly large, despite the absence of claims.

\section{THE DISTRIBUTION OF AGGREGATE CLAIMS UNDER INFLATIONARY CONDITIONS}

In this section we investigate the distribution of $X^{*}(t)$ under the more specific assumption that it is a compound Poisson variate, the claim number process having a Poisson parameter $\lambda$ and the individual claim size distribution having d.f. $B(\cdot)$ at time zero. The method of obtaining the moment generating function (m.g.f.) of $X^{*}(t)$ is essentially that of ANDREws and BRUNNSTROM $(1976)$, though requiring some generalization since they take $B(\cdot)$ to be the d.f. of a single-point distribution.

Consider the time-interval $(j t / m,(j+1) t / m)$ where $m$ is a very large positive integer and $j$ is an integer between $O$ and $(m-1)$. Because the length of this interval, $t / m$ is small, the Poisson claim number process within it approximates a binomial process with parameters 1 and $\lambda t / m$. Therefore, the m.g.f. of aggregate claim amount in this small interval is:

$$
\begin{aligned}
M_{j}^{*}(u) & =\left(1-\frac{\lambda t}{m}\right)+\frac{\lambda t}{m} \beta(u f(j t / m))+O\left(m^{-2}\right) \\
& =1+\frac{\lambda t}{m}[\beta(u f(j t / m))-1]+O\left(m^{-2}\right),
\end{aligned}
$$

where $\beta(u)$ is the m.g.f. associated with $B(\cdot)$. If the additional assumption of independence of sizes of different claims is made, then the cumulant generating function of $X^{*}(t)$ becomes:

$$
\begin{aligned}
K^{*}(u, t) & =\sum_{j=0}^{m-1} \log \left\{1+\frac{\lambda t}{m}[\beta(u f(j t / m))-1]+O\left(m^{-2}\right)\right\} \\
& =\lambda \sum_{j=0}^{m-1} \frac{\beta(u f(j t / m))-1}{m / t}+O\left(m^{-1}\right) .
\end{aligned}
$$

Letting $m \rightarrow \infty$, we see that the c.g.f. becomes:

$$
K^{*}(u, t)=\lambda t\left[\frac{1}{t} \int_{0}^{t} \beta(u f(s)) d s-1\right] .
$$


From this it follows that the $j$-th cumulant of $X^{*}(t)$ is

$$
x_{j}^{*}(t)=\lambda t \alpha_{j}\left\{\frac{1}{t} \int_{0}^{t}[f(s)] j d s\right\},
$$

where $\alpha_{j}$ is the $j$-th order moment (about the origin) of the d.f. $B(\cdot)$ and the second factor on the right is the average value of $[f(s)]^{j}$ over $s \in[0, t]$.

Obviously, the m.g.f. of $X^{*}(t)$ is:

$$
M^{*}(u, t)=\exp \left\{\lambda t\left[\frac{1}{t} \int_{0}^{t} \beta(u f(s)) d s-1\right]\right\}
$$

In the most important special case, $f(s)=e^{k s},(13)$ and (14) can be put in a sometimes more convenient form. Equation (14) becomes:

$$
x_{j}^{*}(t)=\lambda \alpha_{j}\left(e^{j k t}-1\right) / j k,
$$

whence

$$
\begin{aligned}
K^{*}(u, t) & =(\lambda / k)\left[\sum_{j=1}^{\infty} \frac{\alpha_{j}}{j !} \frac{\left(u e^{k t}\right)^{j}}{j}-\sum_{j=1}^{\infty} \frac{\alpha_{j}}{j !} \frac{u^{j}}{j}\right] \\
& =(\lambda / k) \int_{u}^{u e^{k t}} \frac{\beta(v)-1}{v} d v
\end{aligned}
$$

7. AN UPPER BOUND ON THE PROBABILITY OF RUIN UNDER INFLATIONARY CONDITIONS

An upper bound on the ruin probability can be found using the method of Gerber (1973). Define $Y^{*}(t)=Z^{*}(t)-Z^{*}(0)$. Gerber shows that, if $\psi^{*}(x, t)$ is the probability of ruin before time $t$ (in the model of Section 6), then

$$
\psi^{*}(x, t) \leqslant \min _{r} e^{-r x} \max _{0 \leqslant s \leqslant t} E\left[\exp \left\{-r Y^{*}(s)\right\}\right],
$$

where for the sake of simplicity we are now assuming that time has been so scaled that expected number of claims for unit time, i.e. $\lambda$, is equal to unity.

In our case this reduces to:

$$
\psi^{*}(x, t) \leqslant \min _{r} e^{-r x} \max _{0 \leqslant s \leqslant t} \exp \left[-r C^{*}(s)+K^{*}(r, s)\right]
$$

Let us examine the square-bracketed term in (19), By (14), it is

$$
s\left\{-r \cdot \frac{c}{s} \int_{0}^{s} f(u) d u+\lambda \sum_{j=1}^{\infty} r^{j} \alpha_{j / j} \cdot \frac{1}{s} \int_{0}^{\infty}[f(u)]^{j} d u\right\},
$$

where $c$ is premium income per unit time. 
Since all claims are $>0$, the $\alpha_{j}$ 's are all $>0$. Thus for large $r$, the higher powers of $r$ dominate and expression (20) is positive and increasing. It also has a zero at $r=0$. Differentiation (with $s$ constant) shows that it has one turning point. Thus expression (20) is o at $r=0$, becomes negative as $r$ increases, and for $s$ constant has a single real positive zero $\pi(s)$.

For $r>\pi(s)$ it is positive and increasing. In view of this, we can deduce from (18) that:

$$
\psi^{*}(x, t) \leqslant \min _{r \geqslant 0} e^{-r x} \max \left\{1, \exp \left[-r C^{*}(t)+K^{*}(r, t)\right]\right\},
$$

since, for given $r \geqslant 0$, the maximum in (19) is 1 if $r \leqslant \pi(t)$, and is $-r C^{*}(t)+$ $K^{*}(r, t)$ if $r \geqslant \pi(t)$. Note that, in (21) we consider only $r \geqslant 0$. This is because the maximum in (19) is always at least 1 (whether $r$ is positive or negative), so that consideration of $r<0$ tells us no more than that $\psi^{*}(x, t) \leqslant \exp (-r x)$ which is $\geqslant 1$ and can be improved upon by choosing $r=0$ in (19). We can simplify (2I) a little further by noting that the exponential term there is $<1$ when $0<r<\pi(t)$, and so

$$
\psi^{*}(x, t) \leqslant \min _{r \geqslant \pi(t)} \exp \left[-r x-r C^{*}(t)+K^{*}(r, t)\right] .
$$

where we recall that $r=\pi(t)$ is the unique real and positive solution of:

$$
-r C^{*}(t)+K^{*}(r, t)=0 .
$$

The similarity between this result and Gerber's (19), both derived from (18) by very similar reasoning, is to be noted. The two formulas are easily seen to be identical if $f(t)=1$ for all $t$.

\section{Remark}

GERBER (1973, p. 210) commented for the case $f(t)=1$ that inequality (22) is rather sharp if $t$ is not too small. It would follow then in our case of more general $f(t)$ that we could take the right side of inequality (22) as reasonable provided $t$ is not too small and the rate of inflation underlying $f(t)$ is not too large.

In the case of an experience-rated scheme, the whole analysis goes through as before except that $Y^{*}(t)$ is replaced by:

$$
\hat{Y}(t)=Y^{*}(t)+x\left(e^{k t}-1\right) .
$$

Making this replacement and following through the previous working, we soon find that:

$$
\hat{\psi}(x, t) \leqslant \min _{r \geqslant \hat{A}(t)} \exp \left[-r x e^{k t}-r \hat{C}(t)+\hat{K}(r, t)\right]
$$


where $\hat{C,} \hat{K}$ denote $C^{*}, K^{*}$ with constant inflation rate $k$, and $r=\hat{\pi}(t)$ is the unique real and positive solution of the equation

$$
-r x\left(e^{k t}-1\right)-r \hat{C}(t)+\hat{K}(r, t)=0 .
$$

\section{AN APPROXIMATION}

It would be useful to have on hand a simple approximation to the ratio $\psi_{2}^{*}(x, t) / \psi_{1}^{*}(x, t)$ where $\psi_{i}^{*}$ is the ruin probability associated with inflation factor $f_{i}$. Table 1 in Section Io shows that inequality (22) is not always as sharp as we would like, but that the ratio $\psi_{2}^{*}(x, t) / \psi_{1}^{*}(x, t)$ is usually approximated reasonably by the ratio of the upper bounds given by (22). At least this tends to be so in the "interesting" cases where probability of ruin is not too high.

This is demonstrated in Table 2 of Section 10.

\section{INFLATION AND EXPERIENCE RATING COMBINED}

There is no difficulty in combining an inflation factor of $f(t)$ and an experience rating factor of $k$. It is easily checked that reserves at time $t$ are:

$$
x+e^{-k t} \int_{0}^{t} c f(s) e^{k s} d s-e^{-k t} \sum_{i=1}^{N(t)} S_{i} f\left(t_{i}\right) e^{k t_{i},}
$$

which leads us to consider the stochastic process,

$$
\hat{Z}^{*}(t)=x e^{k t}+\hat{C}^{*}(t)-\hat{X}^{*}(t),
$$

where $\hat{C}^{*}(t)$ and $\hat{X}^{*}(t)$ are the premium income and claims outgo respectively up to time $t$ under the influence of an inflation factor of $\exp (k t) f(t)$.

\section{NUMERICAL EXAMPLES}

Consider the case in which the time-axis has been scaled in such a way that, in the absence of any inflation, the claim intensity is 1 per unit time. Suppose that money values have also been so scaled that (again in the absence of inflation) the distribution of individual claim size is $\chi_{66}^{2} / 6$, i.e. m.g.f. is $(1-r / 3)^{-3}$. We shall assume constant rates of inflation, i.e. $f(t)=e^{k t}$, and consider the values $k=0, .05$ and .15 . Suppose that the basic premium income is 1.2 per unit time, thus allowing a safety margin of $20 \%$. Then, by (22) and (23),

$$
\psi^{*}(x, t) \leqslant \min _{r \geqslant \pi(t)} \exp \left[-r x-1.2 r \frac{e^{k t}-1}{k}+K^{*}(r, t)\right],
$$

where $r=\pi(t)$ is the real positive solution of

$$
-1.2 r \frac{e^{k t}-1}{k}+K^{*}(r, t)=0 .
$$


In cases where the minimum in (26) is assumed for $r>\pi(t)$, the relevant value of $r$ is that satisfying the equation:

$$
-x-1.2 \frac{e^{k t}-1}{k}+\frac{1}{k}\left[\frac{\beta\left(r e^{k t}\right)-1}{r}-\frac{\beta(r)-1}{r}\right]=0
$$

i.e.

$$
\left(1-\frac{1}{3} r e^{k t}\right)^{-3}-\left(1-\frac{1}{3} r\right)^{-3}=r\left[k x+1.2\left(e^{k t}-1\right)\right] .
$$

Also

$$
\begin{aligned}
K^{*}(r, t) & =\frac{1}{k} \int_{r^{k t}}^{r^{k t}} \frac{\left(1-\frac{1}{3} v\right)^{-3}-1}{v} d v \\
& =\frac{1}{3^{k}} \int_{r}^{r^{k t}}\left[\left(1-\frac{1}{3} v\right)^{-1}+\left(1-\frac{1}{3} v\right)^{-2}+\left(1-\frac{1}{3} v\right)^{-3}\right] d v \\
& =\left.\frac{1}{k}\left[-\log \left(1-\frac{1}{3} v\right)+\left(1-\frac{1}{3} v\right)^{-1}+\frac{1}{2}\left(1-\frac{1}{3} v\right)^{-2}\right]\right|_{v-r} ^{r e^{k t}}
\end{aligned}
$$

We take initial reserves equal to 5 and, for each value of $k$, calculate for various $t$ the upper bound $(22)$ on $\psi^{*}(5, t)$ and the ratio of this bound to the corresponding bound on $\psi(5, t)$. The results are given in Tables 1 and 2 where the values of $\psi^{*}(x, t)$ obtained from a computer simulation are also given. The sample size for each simulated probability was 2400 .

Similar calculations are made for the case of a negative exponential claim size distribution. Equation $(28)$ is replaced by:

$$
\left(1-r e^{k t}\right)^{-1}-(1-r)^{-1}=r\left[k x+1.2\left(e^{k t}-1\right)\right] .
$$

i.e.

$$
r=\frac{1}{2}\left(1+e^{-k t}\right)\left[1-\sqrt{1-4(1-1 / A) e^{-k t}\left(1+e^{-k t}\right)^{-2}}\right]
$$

where

$$
A=1.2+k x\left(e^{k t}-1\right)^{-1}
$$

Also,

$$
K^{*}(r, t)=\frac{1}{k} \log \left\{\frac{1-\gamma}{1-\gamma e^{k t}}\right\} .
$$

Tables 3 and 4 then summarize these calculations. Once again the results of a computer simulation (sample size again 2400 ) are given.

Several facts emerge from Tables 1 to 4 . 
TABLE 1: UPPER BOUND $(22)$ ON $\psi^{*}(5, t)$ IN CASE OF A $\chi_{6}^{2} / 6$ CLAIM SIZE DISTRIBUTION ${ }^{\mathrm{a}}$

\begin{tabular}{rllllll}
\hline$t$ & \multicolumn{2}{c}{$k=\mathrm{o}$} & \multicolumn{2}{c}{$k=.05$} & \multicolumn{2}{c}{$k=.15$} \\
\hline 1 & .021 & $(.0033)$ & .023 & $(.0038)$ & .031 & $(.0046)$ \\
2 & .057 & $(.0096)$ & .071 & $(.014)$ & .105 & $(.024)$ \\
3 & .094 & $(.023)$ & .122 & $(.031)$ & .194 & $(.048)$ \\
4 & .126 & $(.032)$ & .169 & $(.055)$ & .283 & $(.091)$ \\
5 & .154 & $(.054)$ & .212 & $(.074)$ & .364 & $(.150)$ \\
10 & .235 & $(.098)$ & .360 & $(.172)$ & $.631^{\mathrm{b}}$ & $(.365)$ \\
25 & .273 & $(.165)$ & $.563^{\mathrm{b}}$ & $(.383)$ & $.944^{\mathrm{b}}$ & $(.787)$ \\
$\infty$ & .273 & & $\mathrm{I}^{\mathrm{b}}$ & & \multicolumn{1}{c}{$1^{\mathrm{b}}$} & \\
\hline
\end{tabular}

a Figures in parentheses are simulated ruin probabilities.

b Values based on $r=\pi(t)$.

TABLE 2: ESTIMATE OF RATIO $\psi^{*}(5, t) / \psi(5, t)$ BY THE RATIO OF THE CORRESPONDING UPPER BOUNDS (22) IN CASE OF A $\chi_{6}^{2} / 6$ CLAIM SIZE DISTRIBUTIONa

\begin{tabular}{rllll}
\hline \multicolumn{1}{c}{$t$} & \multicolumn{2}{c}{$k=.05$} & \multicolumn{2}{c}{$k=.15$} \\
\hline 1 & 1.10 & $(1.15)$ & $1.4^{8}$ & $(1.39)$ \\
2 & 1.25 & $(1.46)$ & 1.84 & $(2.50)$ \\
3 & 1.30 & $(1.35)$ & 2.06 & $(2.09)$ \\
4 & 1.34 & $(1.75)$ & 2.25 & $(2.87)$ \\
5 & 1.38 & $(1.38)$ & 2.36 & $(2.79)$ \\
10 & 1.53 & $(1.76)$ & 2.69 & $(3.72)$ \\
25 & 2.06 & $(2.33)$ & 3.46 & $(4.78)$ \\
\hline
\end{tabular}

a Figures in parentheses are taken from computer simulation.

TABLE 3: UPPER BOUND (22) ON $\psi^{*}(5, t)$ IN CASE OF A NEGATIVE EXPONENTIAL CLAIM SIZE DISTRIBUTIONa

\begin{tabular}{rcccccc}
\hline$t$ & \multicolumn{2}{c}{$k=0$} & \multicolumn{2}{c}{$k=.05$} & $k=.15$ \\
\hline 1 & .108 & $(.009)$ & .117 & $(.011)$ & .136 & $(.017)$ \\
2 & .182 & $(.035)$ & .205 & $(.041)$ & .258 & $(.053)$ \\
5 & .311 & $(.096)$ & .379 & $(.121)$ & .529 & $(.200)$ \\
10 & .397 & $(.158)$ & .520 & $(.233)$ & .883 & $(.436)$ \\
\hline
\end{tabular}

a Figures in parentheses are simulated ruin probabilities.

TABLE 4: ESTIMATE OF RATIO $\psi^{*}(5, t) / \psi(5, t)$ BY THE RATIO OF THE CORRESPONDING UPPER BOUNDS (22) IN CASE OF A NEGATIVE EXPONENTIAL CLAIM SIZE DISTRIBUTION ${ }^{\mathrm{a}}$

\begin{tabular}{rcccc}
\hline \hline$t$ & \multicolumn{2}{c}{$k=.05$} & \multicolumn{2}{c}{$k=.15$} \\
\hline 1 & 1.08 & $(1.22)$ & 1.26 & $(1.89)$ \\
2 & 1.13 & $(1.17)$ & 1.42 & $(1.51)$ \\
5 & 1.22 & $(1.26)$ & 1.70 & $(2.08)$ \\
10 & 1.31 & $(1.47)$ & 2.22 & $(2.76)$ \\
\hline
\end{tabular}

a Figures in parentheses are taken from computer simulation. 
Firstly, in Tables 1 and 3 we see that the upper bound (22), even in the case of no inflation, is not as sharp as one might expect after a perusal of the calculations of GERBER (1973, p. 210). The bound does, however, improve with increasing $t$, whether inflation is present or not.

Secondly, for a given pair of inflation rates the ratio of upper bounds (22), as exemplified in Tables 2 and 4 , can serve as a rough approximation to the ratio of the corresponding ruin probabilities, provided that these probabilities are not too large. Even though the simulated results of Tables 1 to 4 are based upon 2400 trials, the simulated low probabilities are still subject to random disturbance. However, for $k=.05$ in Table 2 , the average relative error in the approximation to $\psi^{*}(5, t) / \psi(5, t)$ is $11 \%$. The corresponding figure for $k=.15$ is $15 \%$. If for $k=.15$, this error is calculated only on the basis of those $t$ for which simulated probability is less than .2 (this corresponds to considering the values $t=1,2,3,4,5$ for $k=.05)$, then the average relative error is again only $10 \%$.

In Table 4, the average relative error in the ratio for $t=1,2$ is $8 \%$ for $k=.05$. It is larger for $k=.15$ but mainly as a result of random error at $t=1$ in the simulation.

Thirdly, as $\psi^{*}(5, t)$ increases with increasing $t$, the approximation to $\psi^{*}(5, t) /$ $\psi(5, t)$ dealt with in Tables 2 and 4 becomes poorer.

In summary, it is fair to say that this approximation seems reasonable for $\psi^{*}(5, t)<$ about .2, but thereafter is rather dubious. However, the range $\psi^{*}(5, t)<.2$ is certainly the most interesting from a practical viewpoint.

\section{EXPONENTIAL INFLATION MAKES ULTIMATE RUIN CERTAIN}

The values of 1 given by (22) in the case $t=\infty$ are rather conspicuous in Table 1 , and raise the question of whether ultimate ruin always occurs with probability 1 when inflation is present.

We consider here the case where there exists a constant $K>0$ such that

$$
\int_{0}^{t} f(s) d s \leqslant K f(t) \text { for all } t
$$

For example, if there is a constant rate of inflation, i.e. the inflation factor is exponential, then (29) is satisfied. We also assume that the uninflated premium income is always received at a rate of $c$ per unit time, and that individual claims in excess of $c K$ occur with nonzero probability.

Under these conditions the rather discomforting answer to our question is that no matter how large the initial reserves, no matter how large the safety margin in premiums, no matter how small the rate of inflation (subject to (29)), the ultimate probability of ruin is always 1 .

This is easily proved. Suppose that our assertion is untrue; then $\psi^{*}(x, t)$ approaches a limit $(<1)$ as $t \rightarrow \infty$. 
Then

$$
\left[\frac{d}{d t} \psi^{*}(x, t)\right] /\left[1-\psi^{*}(x, t)\right] \rightarrow 0 \text { as } t \rightarrow \infty
$$

Now let $G(x, t, y)$ denote the probability that an insurer with initial reserve $x$ will survive to time $t$ and have reserves between $o$ and $y$ at that time.

If $B_{t}($.$) denotes the d.f. of individual claim size at time t$, then:

$$
\lambda \int_{0}^{\infty}\left[1-B_{t}(y)\right] d_{y} G(x, t, y) / \int_{0}^{\infty} d_{y} G(x, t, y) \rightarrow 0 \text { as } t \rightarrow \infty
$$

But reserves at time $t$ are at most:

$$
x+c \int_{0}^{t} f(s) d s \leqslant x+c K f(t)
$$

By (31) and (32):

$$
\begin{aligned}
& \lambda \int_{0}^{\infty}\left[1-B_{t}(x+c K f(t))\right] d y G(x, t, y) / \int_{0}^{\infty} d_{y} G(x, t, y) \\
& \leqslant \lambda \int_{0}^{\infty}\left[1-B_{t}(y)\right] d_{y} G(x, t, y) / \int_{0}^{\infty} d_{y} G(x, t, y) \\
& \rightarrow 0 \text { as } t \rightarrow \infty
\end{aligned}
$$

i.e.

$$
1-B_{t}(x+c K f(t)) \rightarrow 0 \text { as } t \rightarrow \infty
$$

But, of course

$$
B_{t}(z)=B(z / f(t))
$$

so that (33) becomes:

i.e.

$$
1-B(c K+x / f(t)) \rightarrow 0 \text { as } t \rightarrow \infty \text {. }
$$

$$
1-B(c K)=0 .
$$

Since this contradicts our assumption that larger claims than $c K$ (uninflated) can occur, our hypothesis of $\psi^{*}(x, t)<1$ is false.

By an identical line of reasoning, we find that if individual claims in excess of $x+c K$ can occur in an experience rated scheme, then the probability of ultimate ruin is 1 . This result was conjectured (though without any condition on the distribution of individual claim sizes) by Sidney Benjamin.

As was remarked in Section 5, this result is not entirely intuitive. However, it does become reasonable when one notes that (by formula (7)), the contribution to reserves at time $t$ of all safety margins paid up to then is

$$
\begin{aligned}
& (1+\eta)^{-1} \eta c e^{-k t} \int_{0}^{s} e^{k s} d s \\
& =(\eta c / k)\left(1-e^{-k t}\right) /(1+\eta)
\end{aligned}
$$


where $\eta$ is the proportion of risk premium taken as a safety margin. We see that accumulated safety margins converge to a finite limit with increasing $t$, i.e. the average safety margin per unit time tends to zero. In these circumstances, it is not surprising that $\hat{\psi}(x, \infty)=1$.

This suggests that the experience rating formula (5) should be replaced by one which does not refund most safety margin. Perhaps, we could take

$$
d C(t)=\left\{c-k\left[\frac{C(t)}{1+\eta}-X(t)\right]\right\} d t .
$$

i.e. only the risk premium $C(t) /(I+\eta)$ is allowed for in the experience rating. Thus (34) can be rewritten as

$$
d C(t)=\left\{\frac{\eta c}{1+\eta}+\left\{\frac{c}{1+\eta}-k\left[\frac{C(t)}{1+\eta}-X(t)\right]\right\}\right\} d t,
$$

and we can see that a constant rate of safety margin $c \eta /(1+\eta)$ is maintained in addition to the experience rated risk premium.

However, there may be some sales difficulties with rating formula (34), since the proportion of the premium absorbed by the safety margin increases as the claims experience improves. One can well imagine the insured objecting to an increase in the relative safety margin being occasioned by a favourable experience.

\section{ALLOWANCE FOR EARNINGS ON ASSETS}

Of course, all of the preceding analysis has been made on the assumption that the free reserves of the insurer earn no interest. We now relax this assumption and suppose that interest is earned at a rate such that a unit invested at time zero accumulates to amount $A(t)$ at time $t$. Then the free reserves at time $t$ under the operation of both interest and inflation are:

$$
x A(t)+\int_{0}^{t}(f(s) A(t) / A(s)) d C(s)-\int_{0}^{t}(f(s) A(t) / A(s)) d X(s)
$$

Discounting these free reserves back to time zero, we obtain

$$
x+\int_{0}^{t}(f(s) / A(s)) d C(s)-\int_{0}^{t}(f(s) / A(s)) d X(s),
$$

so that a process subject to an inflation factor $f(t)$ and an interest accumulation factor $A(t)$ is equivalent to a process with just an inflation factor of $f(t) / A(t)$. What matters, therefore, is whether rate of inflation is greater or less than the rate of interest. For example, if the difference between the force of inflation and the force of interest is constant and positive (be it ever so small), then the result of Section 11, viz. unit probability of ruin, still holds. 


\section{REFERENCES}

Andrews, J. G. and R. R. L. Brunnstrom (1976). The discounted cost of a random sequence of failures, Bulletin of the Institute of Mathematics and its Applications, 12, $275-279$.

CRAMÉR, H. (1930). On the mathematical theory of risk, Skandia Jubilee Volume, Stockholm.

CRAMER, H. (1955). Collective risk theory, a survey of the theory from the point of view of the theory of stochastic processes, Skandia Jubilee Volume, Stockholm.

Gerber, H. U. (1973). Martingales in risk theory, Mitteilungen der Vereinigung schweizerischer Versicherungsmathematiker, 73, 205-216.

LundBerg, F. (1909). Uber die Theorie der Rückversicherung, Transactions of the 6th International Congress of Actuaries, 1, 877-948.

SEAL, H. L. (1969). Simulation of the ruin potential of nonlife insurance companies, Transactions of the Society of Actuaries, 21, 563-585. 